\title{
An appraisal of clinical practice guidelines for constipation: a right attitude towards to guidelines
}

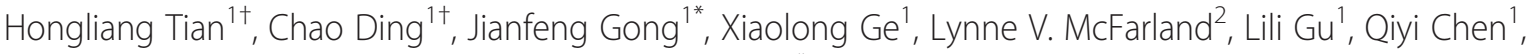 \\ Chunlian Ma', Weiming Zhu', Jieshou Li and Ning Li ${ }^{1 *}$
}

\begin{abstract}
Background: Clinical practice guidelines (CPGs) are formally developed statements that assist users to provide proper health care for a kind of disease and play a significant contribution in healthcare system. This study report the methodological quality of CPGs on constipation.

Methods: The "Appraisal of Guidelines and Research and Evaluation" (AGREEII) instrument was developed to determine the quality of CPGs. A comprehensive search was developed using five databases and three guideline websites until/up to December, 2015. Four independent authors evaluated the methodological issues of the CPGs by the AGREEll instrument.

Results: We identified 22 relevant guidelines on constipation from 1234 citations. The overall agreement among evaluators was 0.84 using the intra-class correlation coefficient. The mean AGREEll scores for the domains "scope and purpose" (51.77) and "rigor of development" (56.73) were moderate; afterward, three domains "stakeholder involvement" (32.23), "editorial independence" (29.59) and "applicability" (29.14) were low scores. The "clarity and presentation" (23.73) had the lowest scores.

Conclusion: Although existing constipation guidelines may accurately reflect current clinical practices, many guidelines' methodological quality is low. Therefore, more emphasis and attentions should be taken to the development of high-quality guidelines.
\end{abstract}

Keywords: Constipation, Clinical practice guideline, AGREEII

\section{Background}

Constipation is a disorder defined by incomplete defecation, and/or infrequent bowel movements which associated with persistent difficult and/or painful defecation, fecal incontinence, and abdominal pain [1]. It is a common clinical functional diseases. The worldwide constipation surveys show a wide range of prevalence rates between $1 \%$ and $>20 \%$ in western populations, although, a recent epidemiological reports found $16 \%$ general adult populations were constipation [2]. Constipation may be found for up to $20 \%$ of community-

\footnotetext{
* Correspondence: gongjianfeng@aliyun.com; liningrigsnju@163.com Tian Hongliang and Ding Chao are joint first authors.

${ }^{\dagger}$ Equal contributors

'Department of General Surgery, Jinling Hospital, Medical School of Nanjing University, No. 305 East Zhongshan Road, Nanjing 210002, China

Full list of author information is available at the end of the article
}

dwelling elderly individuals. Moreover the incidence of functional constipation in childhood estimated $3 \%$ [3].

Because of its high disease burden, the treatment of constipation has become an important issue for clinicians and patients. During the last two decade there were more than 20 developed clinical practice guidelines (CPGs) to manage the constipation. The main role of the CPGs is to give clear recommendations to help clinicians make appropriate clinical decision for specific clinical circumstance $[4,5]$.

However, not all guidelines are developed with the same methodologically rigorous approaches, there is no research for evaluating the quality of CPGs on constipation so far. With the above in mind, the objectives of the present study was to systematically review guidelines 
using the appraisal of guidelines for research and evaluation (AGREEII) instrument related to constipation [6].

\section{Methods}

Literature search

An electronic literature search using multiple databases (PubMed, EMBASE, The China Journal Full-text Database, Chinese Biomedical Literature Database, Chinese Scientific Journals Full-text Database), and guideline website or databases-including the Guidelines International Network (GIN) Database, the National Guideline Clearinghouse (NGC), National Institute for Health and Care Excellence (NICE), Scottish Intercollegiate Guidelines Network (SIGN), National Comprehensive Cancer Network (NCCN) was conducted limited to Chinese and English from the inception to May 2015. $\mathrm{MeSH}$ terms and text words "guideline, consensus, recommendation, criteria, statement, constipation" for constipation and guidelines were used within the MEDLINE database. The same search strategy was made applicable for the other databases or websites.

\section{Guideline selection and data extraction}

Four reviewers (THL,DC,LN,GJF) independently extracted the guidelines which met the characteristics (for example, a clear guideline definition as proposed by the institute of Medicine [4], focused exclusively on constipation disease). We constructed a standard form table to extract the data of guidelines. Four reviewers extracted data separately, disagreements were discussed or by a fifth reviewer (GXL) if no consensus was reached.

\section{Quality appraisal and recommendation}

We evaluated the twenty-two included CPGs quality by AGREEII instrument [6]. The instrument includes a 23item tool comprising six quality domains. The four authors read the entire AGREEII handbook and then independently rated all included guidelines using formula as follows:

$$
\frac{\text { Obtained score }- \text { Minimum possible score }}{\text { Maximum possible socre }- \text { Minimum possible score }} \times 100 \%
$$

According to the handbook for use of the AGREEII instrument, the six domain scores were considered independently. Finally, a guideline is labelled as "strongly recommended" if most domain scores are greater than $60 \%$. Guideline is "recommended" when most scores are between $30 \%$ and $60 \%$. A guideline is labelled as "not recommended" when most domain scores are less than $30 \%$ [7].

\section{Statistical analysis}

A descriptive study of item frequency was carried out and the AGREEII domain scores calculated as means.
Intra-class correlation coefficients (ICCs) is a measure of the reliability of measurements or ratings within each domain [8]. Statistically significant was considered if $p$ value less than 0.05 .

\section{Results and discussion}

\section{Literature search}

Figure 1 shows how we screened the guidelines, we preliminary search found 1234 citations, 35 were excluded because they were duplicate citations. By screening their titles and abstracts and 1,146 citations were ineligible as they didn't meet the characteristics of constipation CPGs, 31 articles were excluded from the left 53 studies as following: eight were duplicates, seven were not in English or Chinese, 14 were guidelines not related to constipation, and 2 guidelines were the old version. Finally, a total of 22 guidelines were included [9-31].

\section{Guideline characteristics}

The summary of CPGs baseline data were shown in Table 1. The twenty-two CPGs published between 2000 and 2014. Of the 22 selected CPGs, half of were from north America (America and Canada), six from European (UK, Ireland, Italy, Sweden) and the remaining five were from Asia (two from China, one from Korea, one from Indonesia and one multi-national),respectively. The scope of the CPRs varied: one guideline topic covered prevention, diagnosis and treatment of constipation [31]; two focused only on prevention and treatment [14, 16]; 16 covered diagnosis and treatment $[9,12,13,15,17-19,21-29]$; three only focused on treatment $[10,20,30]$ and one focused on prevention [11]. CPGs cited a range of number of references (range: 0-364, mean: 78) and were of varying length (mean number of pages $=25$, range: $5-255$ ). Each of the domains being evaluated using the AGREEII appraisal (Table 2). The ICCs score was moderate among raters (0.84; 95 \% CI, 0.56-0.86).

\section{Appraisal of guidelines Domain 1}

Scope and purpose is concerned with the overall aim of the guideline, the specific health questions, and the target population (items 1-3) [32]. This domain's mean score was $51.77 \%$, and nine of the guidelines (47.62 \%) scored below $50 \%$ [9, 10, 12, 21, 22, 25, 26, 29, 31].

\section{Domain 2}

Stakeholder involvement focuses on the extent to which the guideline was developed by the appropriate stakeholders and represents the views of its intended users (items 4-6) [32]. Of all AGREEII domains, this domain received the lowest scores (23.73 \%) with only one CPG scoring over $50 \%$. 


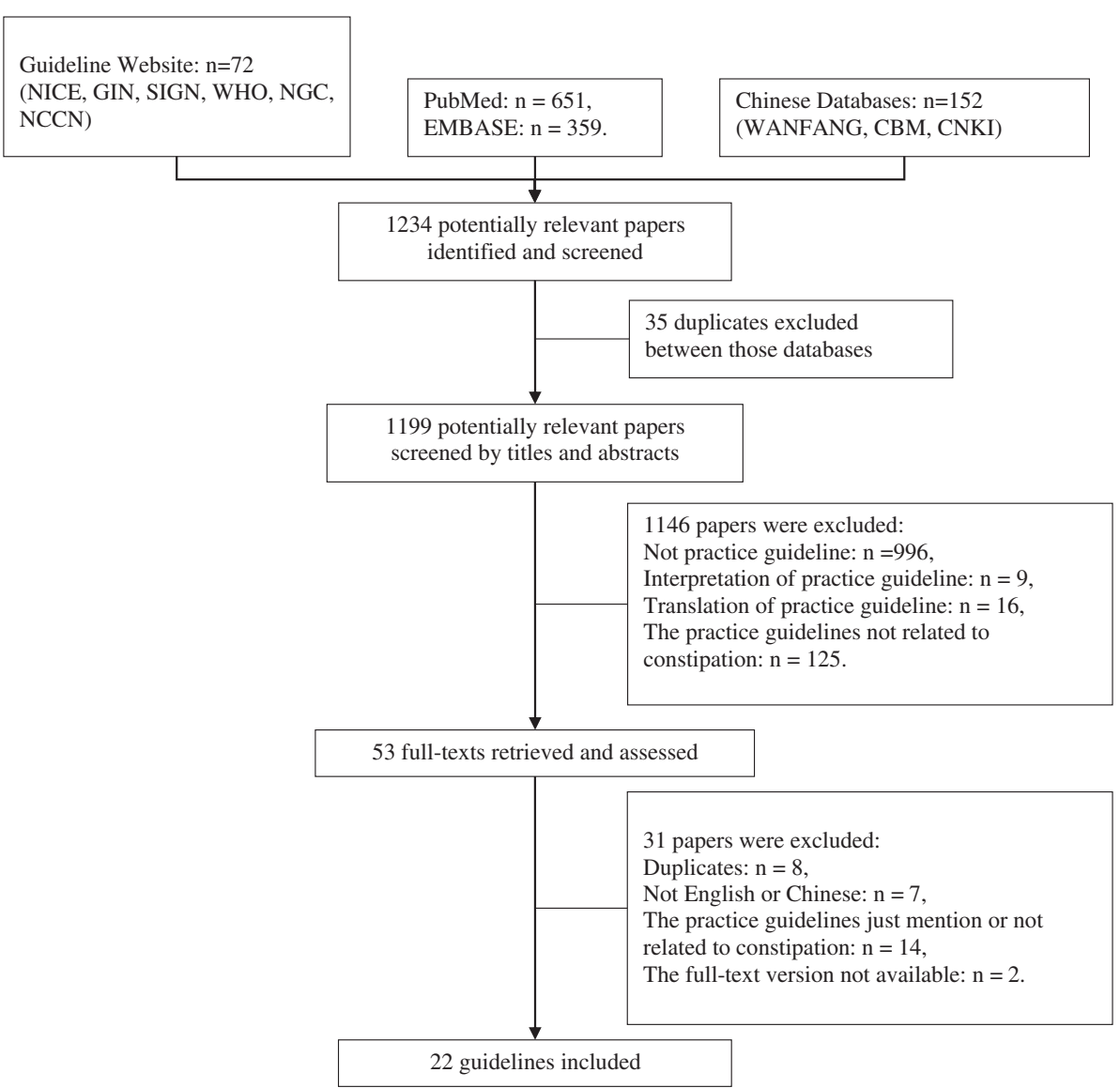

Fig. 1 Flow of information through the different phases of the literature search

Eighteen CPGs had been developed by a multidisciplinary organization $(81.82 \%)[9,11,13-30]$.

\section{Domain 3}

Rigor of development criteria relates to the process used to gather and synthesize the evidence, the methods to formulate the recommendations, and to update those (items 7-14) [32]. Overall, the mean score for this domain was only $32.23 \%$ (range, $3 \%$ to $66 \%$ ), with 18 CPGs scoring $<50 \%$. Meanwhile, only five CPGs reported systematic evidence searching [12, 16, 18, 27, 30], and Just $40.90 \%(9 / 22)$ guidelines provided the methods for formulating the recommendations [11-16, 26, 27, 29]. Moreover, an explicit link between the recommendations and the evidence were explicit in 20/22 of the guidelines and only five guidelines described a procedure about updating $[11,13,21,27,29]$.

\section{Domain 4}

Clarity of presentation deals with the language, structure, and format of the guideline (items 15-17) [32]. The mean score for this domain was $56.73 \%$ (range, $36 \%$ to $83 \%)$. Most CPGs provided a concrete and precise description of key recommendations with only eight guidelines scoring less than $50 \%[9,10,14,22$, $25,26,30,31]$.

\section{Domain 5}

Applicability pertains to the likely barriers and facilitators to implementation, strategies to improve uptake, and resource implications of applying the guideline (items 18-21) [32]. This domain's score was $29.14 \%$ (range, $10 \%$ to $58 \%$ ) and only three CPGs scored $>50 \%[11,18,20]$. A total of 10 CPGs discussed barriers to implementing the guideline's recommendations $[11,13,14,16,18,20,21,29-31]$ and 7 guideline provides advice and/or tools on how the recommendations can be put into practice $[11,16,18,20,29-31]$. Resource implications were not explicitly discussed, only five CPGs offered cost implications [11, 18, 20, 29, 30].

\section{Domain 6}

Editorial independence is concerned with the formulation of recommendations not being unduly biased with competing interests (items 22-23) [32]. The mean score for this domain was $29.59 \%$. Fifteen guidelines scored 
Table 1 Characteristics of clinical practice guidelines for constipation

\begin{tabular}{|c|c|c|c|c|c|c|c|c|c|c|c|c|}
\hline Title & Year & Country & Organization & $\begin{array}{l}\text { Type of } \\
\text { constipation }\end{array}$ & $\begin{array}{l}\text { Topics } \\
\text { covered }\end{array}$ & Update & $\begin{array}{l}\text { No. of } \\
\text { reference }\end{array}$ & $\begin{array}{l}\text { Guideline } \\
\text { page }\end{array}$ & $\begin{array}{l}\text { Publication } \\
\text { types }\end{array}$ & $\begin{array}{l}\text { Systematic } \\
\text { search }\end{array}$ & $\begin{array}{l}\text { Evidence- } \\
\text { based }\end{array}$ & Financial \\
\hline $\begin{array}{l}\text { American Gastroenterological } \\
\text { Association Medical Position } \\
\text { Statement: Guidelines on } \\
\text { Constipation [9] }\end{array}$ & 2000 & USA & AGA & $\begin{array}{l}\text { Slow-Transit } \\
\text { Constipation, Pelvic } \\
\text { Floor Dysfunction, } \\
\text { Combination } \\
\text { Syndromes }\end{array}$ & $\begin{array}{l}\text { Diagnosis, } \\
\text { treatment }\end{array}$ & $\begin{array}{l}\text { Not } \\
\text { reported }\end{array}$ & 1 & 6 & Journal & $\begin{array}{l}\text { Not } \\
\text { reported }\end{array}$ & $\begin{array}{l}\text { Not } \\
\text { reported }\end{array}$ & Not reported \\
\hline $\begin{array}{l}\text { Practice Guidelines for the } \\
\text { Management of Constipation } \\
\text { in adults [10] }\end{array}$ & 2002 & USA & Not reported & $\begin{array}{l}\text { Constipation in } \\
\text { adults }\end{array}$ & Treatment & $\begin{array}{l}\text { Not } \\
\text { reported }\end{array}$ & 203 & 51 & $\begin{array}{l}\text { Special website } \\
\text { (http:// } \\
\text { www.arna.com.au/) }\end{array}$ & $\begin{array}{l}\text { Not } \\
\text { reported }\end{array}$ & $\begin{array}{l}\text { Not } \\
\text { reported }\end{array}$ & Not reported \\
\hline $\begin{array}{l}\text { Prevention of constipation in } \\
\text { the older adult population [11] }\end{array}$ & 2005 & Canada & RNAOPA & $\begin{array}{l}\text { Older adult } \\
\text { populations }\end{array}$ & Prevention & Yes & 226 & 16 & $\begin{array}{l}\text { Special website } \\
\text { (http://rnao.ca/bpg/ } \\
\text { guidelines/ } \\
\text { prevention- } \\
\text { constipation-older- } \\
\text { adult-population) }\end{array}$ & $\begin{array}{l}\text { Not } \\
\text { reported }\end{array}$ & Yes & Not reported \\
\hline $\begin{array}{l}\text { Management of chronic } \\
\text { constipation: } \\
\text { recommendations from } \\
\text { a consensus panel [12] }\end{array}$ & 2005 & USA & None & $\begin{array}{l}\text { Chronic } \\
\text { constipation }\end{array}$ & $\begin{array}{l}\text { Diagnosis, } \\
\text { treatment }\end{array}$ & $\begin{array}{l}\text { Not } \\
\text { reported }\end{array}$ & 55 & 8 & Journal & Yes & $\begin{array}{l}\text { Not } \\
\text { reported }\end{array}$ & $\begin{array}{l}\text { Yes, } \\
\text { Pharmaceuticals } \\
\text { Cor. }\end{array}$ \\
\hline $\begin{array}{l}\text { Evaluation and Treatment of } \\
\text { Constipation in Infants and } \\
\text { Children: Recommendations } \\
\text { of the North American } \\
\text { Society for Pediatric } \\
\text { Gastroenterology, Hepatology } \\
\text { and Nutrition [13] }\end{array}$ & 2006 & USA & $\begin{array}{l}\text { NASPGHAN } \\
\text { Constipation } \\
\text { Guideline } \\
\text { Committee }\end{array}$ & $\begin{array}{l}\text { Infants and } \\
\text { Children } \\
\text { constipation }\end{array}$ & $\begin{array}{l}\text { Diagnosis, } \\
\text { treatment }\end{array}$ & Yes & 96 & 13 & Journal & $\begin{array}{l}\text { Not } \\
\text { reported }\end{array}$ & Yes & Not reported \\
\hline $\begin{array}{l}\text { Putting evidence into } \\
\text { practice:evidence-based } \\
\text { inventions for the prevention } \\
\text { and management of } \\
\text { constipation in patients with } \\
\text { cancer [14] }\end{array}$ & 2008 & USA & ONS & $\begin{array}{l}\text { constipation in } \\
\text { patients with } \\
\text { cancer }\end{array}$ & $\begin{array}{l}\text { Prevention, } \\
\text { treatment }\end{array}$ & $\begin{array}{l}\text { Not } \\
\text { reported }\end{array}$ & 72 & 21 & Journal & $\begin{array}{l}\text { Not } \\
\text { reported }\end{array}$ & Yes & Not reported \\
\hline $\begin{array}{l}\text { The management of } \\
\text { constipation in palliative } \\
\text { care: clinical practice } \\
\text { recommendations [15] }\end{array}$ & 2008 & Ireland & $\begin{array}{l}\text { The European } \\
\text { Consensus Group } \\
\text { on Constipation } \\
\text { in Palliative Care }\end{array}$ & Constipation & $\begin{array}{l}\text { Diagnosis, } \\
\text { treatment }\end{array}$ & $\begin{array}{l}\text { Not } \\
\text { reported }\end{array}$ & 43 & 13 & Journal & $\begin{array}{l}\text { Not } \\
\text { reported }\end{array}$ & Yes & $\begin{array}{l}\text { Yes, } \\
\text { Pharmaceuticals } \\
\text { Cor. }\end{array}$ \\
\hline $\begin{array}{l}\text { Management of } \\
\text { constipation [16] }\end{array}$ & 2009 & UK & NICE & Older adults & $\begin{array}{l}\text { Prevention, } \\
\text { treatment }\end{array}$ & $\begin{array}{l}\text { Not } \\
\text { reported }\end{array}$ & 34 & 10 & $\begin{array}{l}\text { Special website } \\
\text { (AHRQ) }\end{array}$ & Yes & Yes & Not reported \\
\hline $\begin{array}{l}\text { National Consensus on } \\
\text { The Management of } \\
\text { Constipation in Indonesia }\end{array}$ & 2010 & Indonesia & ISG & Constipation & $\begin{array}{l}\text { Diagnosis, } \\
\text { treatment }\end{array}$ & $\begin{array}{l}\text { Not } \\
\text { reported }\end{array}$ & 18 & 8 & Journal & $\begin{array}{l}\text { Not } \\
\text { reported }\end{array}$ & No & Not reported \\
\hline
\end{tabular}

Constipation in Indonesia

$2010[17]$ 
Table 1 Characteristics of clinical practice guidelines for constipation (Continued)

Constipation in Children and Young People:

Diagnosis and Management

of Idiopathic Childhood

Constipation in Primary
and Secondary Care [18]

Consensus Recommendations 2010 Canada

for the Management of

Constipation in Patients with

Advanced, Progressive

Illness [19]

Prucalopride for the treatment 2010

of chronic constipation in

women [20]

Common views on diagnosis 2011 China and treatment of chronic

constipation with Chinese

medicine [21]

World Gastroenterology

Organisation Global Guideline

Constipation-A Global

Perspective [22]

Consensus statement AlGO/

SICCR diagnosis and treatment

of chronic constipation and

obstructed defecation [23, 24]

Practical Treatments for

Constipation in Korea [25]

Diagnosis and Treatment

Guideline of Chronic

Constipation in China [26]

Evaluation and Treatment of

Functional Constipation in

Infants and Children: Evidence

BSPGHAN and NASPGIAN From

Primary Care Management

of Chronic Constipation in

Asia: The ANMA Chronic

Constipation Too I [28]

\begin{tabular}{|c|c|c|c|c|c|c|c|c|c|c|c|}
\hline 2010 & UK & NCC-WCH & $\begin{array}{l}\text { Constipation in } \\
\text { children and } \\
\text { young people }\end{array}$ & $\begin{array}{l}\text { Diagnosis, } \\
\text { treatment }\end{array}$ & $\begin{array}{l}\text { Not } \\
\text { reported }\end{array}$ & 140 & 255 & $\begin{array}{l}\text { Special website } \\
\text { (NICE) }\end{array}$ & Yes & Yes & Not reported \\
\hline 2010 & Canada & $\begin{array}{l}\text { The Canadian } \\
\text { Consensus } \\
\text { Development } \\
\text { Group }\end{array}$ & $\begin{array}{l}\text { Patients with } \\
\text { Advanced, } \\
\text { Progressive Illness }\end{array}$ & $\begin{array}{l}\text { Diagnosis, } \\
\text { treatment }\end{array}$ & $\begin{array}{l}\text { Not } \\
\text { reported }\end{array}$ & 20 & 13 & Journal & $\begin{array}{l}\text { Not } \\
\text { reported }\end{array}$ & Yes & $\begin{array}{l}\text { Yes (edu cational } \\
\text { grant from Wyeth) }\end{array}$ \\
\hline 2010 & UK & NICE & $\begin{array}{l}\text { Chronic } \\
\text { constipation in } \\
\text { women }\end{array}$ & Treatment & $\begin{array}{l}\text { Not } \\
\text { reported }\end{array}$ & 11 & 38 & $\begin{array}{l}\text { Special website } \\
\text { (Cancer Care } \\
\text { Ontario Website) }\end{array}$ & $\begin{array}{l}\text { Not } \\
\text { reported }\end{array}$ & Yes & Not reported \\
\hline 2011 & China & CACM & $\begin{array}{l}\text { Chronic } \\
\text { constipation }\end{array}$ & $\begin{array}{l}\text { Diagnosis, } \\
\text { treatment }\end{array}$ & Yes & 24 & 5 & Journal & $\begin{array}{l}\text { Not } \\
\text { reported }\end{array}$ & No & Not reported \\
\hline 2011 & Sweden & WGO & Adult patients & $\begin{array}{l}\text { Diagnosis, } \\
\text { treatment }\end{array}$ & $\begin{array}{l}\text { Not } \\
\text { reported }\end{array}$ & 0 & 5 & Journal & $\begin{array}{l}\text { Not } \\
\text { reported }\end{array}$ & Yes & None \\
\hline 2012 & Italy & $\mathrm{AIGO} / \mathrm{SICCR}$ & $\begin{array}{l}\text { Chronic } \\
\text { constipation and } \\
\text { obstructed } \\
\text { defecation }\end{array}$ & $\begin{array}{l}\text { Diagnosis } \\
\text { Treatment }\end{array}$ & $\begin{array}{l}\text { Not } \\
\text { Reported }\end{array}$ & 364 & 30 & Journal & $\begin{array}{l}\text { Not } \\
\text { reported }\end{array}$ & Yes & $\begin{array}{l}\text { Associazione } \\
\text { Italiana } \\
\text { Gastroenterologi } \\
\text { and Endoscopisti } \\
\text { Digestivi } \\
\text { Ospedalieri }\end{array}$ \\
\hline 2012 & Korea & KSNM & Constipation & $\begin{array}{l}\text { Diagnosis," } \\
\text { treatment }\end{array}$ & $\begin{array}{l}\text { Not } \\
\text { reported }\end{array}$ & 63 & 9 & Journal & $\begin{array}{l}\text { Not } \\
\text { reported }\end{array}$ & $\begin{array}{l}\text { Not } \\
\text { reported }\end{array}$ & Not reported \\
\hline 2013 & China & CMAG/CMAS & $\begin{array}{l}\text { Chronic } \\
\text { constipation }\end{array}$ & $\begin{array}{l}\text { Diagnosis, } \\
\text { treatment }\end{array}$ & $\begin{array}{l}\text { Not } \\
\text { reported }\end{array}$ & 63 & 8 & Journal & $\begin{array}{l}\text { Not } \\
\text { reported }\end{array}$ & $\begin{array}{l}\text { Not } \\
\text { reported }\end{array}$ & Not reported \\
\hline 2013 & USA & $\begin{array}{l}\text { ESPGHAN and } \\
\text { NASPGHAN }\end{array}$ & $\begin{array}{l}\text { Functional } \\
\text { Constipation in } \\
\text { Infants and } \\
\text { Children }\end{array}$ & $\begin{array}{l}\text { Diagnosis, } \\
\text { treatment }\end{array}$ & Yes & 111 & 17 & Journal & Yes & Yes & $\begin{array}{l}\text { NASPGHAN } \\
\text { and ESPGHAN }\end{array}$ \\
\hline 2013 & Asia & ANMA & $\begin{array}{l}\text { Chronic } \\
\text { Constipation }\end{array}$ & $\begin{array}{l}\text { Diagnosis," } \\
\text { treatment }\end{array}$ & $\begin{array}{l}\text { Not } \\
\text { reported }\end{array}$ & 124 & 12 & Journal & $\begin{array}{l}\text { Not } \\
\text { reported }\end{array}$ & No & $\begin{array}{l}\text { educational grant } \\
\text { was receive d } \\
\text { from Janssen } \\
\text { Pharmaceuticals } \\
\text { and Boehringer } \\
\text { Ingelheim }\end{array}$ \\
\hline
\end{tabular}


Table 1 Characteristics of clinical practice guidelines for constipation (Continued)

\begin{tabular}{|c|c|c|c|c|c|c|c|c|c|c|c|c|}
\hline $\begin{array}{l}\text { American Gastroenterological } \\
\text { Association Medical Position } \\
\text { Statement on Constipation [29] }\end{array}$ & 2013 & USA & AGA & $\begin{array}{l}\text { Chronic } \\
\text { Constipation }\end{array}$ & $\begin{array}{l}\text { Diagnosis, } \\
\text { treatment }\end{array}$ & Yes & 1 & 7 & Journal & $\begin{array}{l}\text { Not } \\
\text { reported }\end{array}$ & Yes & Not reported \\
\hline $\begin{array}{l}\text { Lubiprostone for treating } \\
\text { chronic idiopathic } \\
\text { constipation [30] }\end{array}$ & 2014 & UK & NICE & $\begin{array}{l}\text { Chronic } \\
\text { idiopathic } \\
\text { constipation }\end{array}$ & Treatment & $\begin{array}{l}\text { Not } \\
\text { reported }\end{array}$ & 0 & 9 & $\begin{array}{l}\text { Special website } \\
\text { (NICE) }\end{array}$ & Yes & Yes & NICE \\
\hline $\begin{array}{l}\text { Emerging treatments in } \\
\text { neurogastroenterology: a } \\
\text { multidisciplinary working }\end{array}$ & 2014 & USA & None & $\begin{array}{l}\text { Opioid-induced } \\
\text { constipation }\end{array}$ & $\begin{array}{l}\text { Prevention, } \\
\text { diagnosis, } \\
\text { treatment }\end{array}$ & $\begin{array}{l}\text { Not } \\
\text { reported }\end{array}$ & 61 & 10 & Journal & $\begin{array}{l}\text { Not } \\
\text { reported }\end{array}$ & $\begin{array}{l}\text { Not } \\
\text { reported }\end{array}$ & AstraZeneca \\
\hline
\end{tabular}

multidisciplinary working

group consensus statement

on opioid-induced

Footnotes: NICE National Institute for Health and Care Excellence, ESMO European Society for Medical Oncology, ESDO European Society of Digestive Oncology, NCCN National Comprehensive Cancer Network, SEOM Spanish Society of Medical Oncology, GIN Guidelines International Network, NASPGHAN North American Society for Pediatric Gastroenterology, Hepatology and Nutrition, AIGO Italian Association of Hospital

Gastroenterologists, SICCR Italian Society of Colo-Rectal Surgery, NCC-WCH National Collaborating Centre for Women's and Children's Health, KSNM Constipation Study Group in the Korean Society of Neurogastroenterology and Motility, NASPGHAN the North American Society for Pediatric Gastroenterology Hepatology and Nutrition, ESPGHAN: the European Society for Pediatric Gastroenterology, Hepatology, and Nutrition, ANMA the Ology and Motility, NASPGHAN the North American Society for Pediatric Gastroenterology Hepatology and Nutrition, ESPGHAN: the European Society for Pediatric Gastroenterology, Hepatology, and Nutrition, ANMA the Asian Neurogastroenterology and Motility Association, AGA American Gastroenterological Association, RNAOPA Registered Nurses Assocation of Ontario-Professional Association, ONS the Oncology Nursing Society, ISG
The Indonesian Society of Gastroenterology, CACM China Association of Chinese Medicine, WGO World Gastroenterology Organization, CMAG/CMAS Chinese Medical Association of Gastroenterology Branch, Chinese The Indonesian Society of Gastroenterolog 
Table 2 Guideline score according to score on each of the domains assessed by the AGREEll instrument

\begin{tabular}{|c|c|c|c|c|c|c|c|}
\hline Title & $\begin{array}{l}\text { Scope and } \\
\text { Purpose }\end{array}$ & Stakeholder & Rigour & Clarity & Applicability & $\begin{array}{l}\text { Editorial } \\
\text { Independence }\end{array}$ & Recommendation \\
\hline $\begin{array}{l}\text { American Gastroenterological } \\
\text { Association Medical Position } \\
\text { Statement: Guidelines on } \\
\text { Constipation [9] }\end{array}$ & $31 \%$ & $11 \%$ & $7 \%$ & $44 \%$ & $15 \%$ & $0 \%$ & Not recommended \\
\hline $\begin{array}{l}\text { Practice Guidelines for the } \\
\text { Management of Constipation } \\
\text { in adults [10] }\end{array}$ & $44 \%$ & $22 \%$ & $36 \%$ & $44 \%$ & $25 \%$ & $0 \%$ & Not recommended \\
\hline $\begin{array}{l}\text { Prevention of constipation in } \\
\text { the older adult population [1 } 1 \text { ] }\end{array}$ & $81 \%$ & $39 \%$ & $58 \%$ & $72 \%$ & $54 \%$ & $54 \%$ & Strongly recommended \\
\hline $\begin{array}{l}\text { Management of chronic } \\
\text { constipation: recommendations } \\
\text { from a consensus panel [12] }\end{array}$ & $39 \%$ & $14 \%$ & $9 \%$ & $53 \%$ & $17 \%$ & $33 \%$ & Not recommended \\
\hline $\begin{array}{l}\text { Evaluation and Treatment of } \\
\text { Constipation in Infants and } \\
\text { Children: Recommendations } \\
\text { of the North American Society } \\
\text { for Pediatric Gastroenterology, } \\
\text { Hepatology and Nutrition [13] }\end{array}$ & $56 \%$ & $31 \%$ & $46 \%$ & $58 \%$ & $31 \%$ & $0 \%$ & Recommended \\
\hline $\begin{array}{l}\text { Putting evidence into practice: } \\
\text { evidence-based inventions for } \\
\text { the prevention and management } \\
\text { of constipation in patients with } \\
\text { cancer [14] }\end{array}$ & $64 \%$ & $28 \%$ & $45 \%$ & $47 \%$ & $33 \%$ & $0 \%$ & Recommended \\
\hline $\begin{array}{l}\text { The management of constipation } \\
\text { in palliative care: clinical practice } \\
\text { recommendations [15] }\end{array}$ & $56 \%$ & $17 \%$ & $41 \%$ & $67 \%$ & $23 \%$ & $21 \%$ & Not recommended \\
\hline Management of constipation [16] & $75 \%$ & $31 \%$ & $63 \%$ & $67 \%$ & $44 \%$ & $29 \%$ & Recommended \\
\hline $\begin{array}{l}\text { National Consensus on The } \\
\text { Management of Constipation in } \\
\text { Indonesia } 2010 \text { [17] }\end{array}$ & $56 \%$ & $8 \%$ & $3 \%$ & $69 \%$ & $10 \%$ & $0 \%$ & Not recommended \\
\hline $\begin{array}{l}\text { Constipation in Children and } \\
\text { Young People: Diagnosis and } \\
\text { Management of Idiopathic } \\
\text { Childhood Constipation in } \\
\text { Primary and Secondary Care [18] }\end{array}$ & $78 \%$ & $61 \%$ & $50 \%$ & $75 \%$ & $54 \%$ & $71 \%$ & Strongly recommended \\
\hline $\begin{array}{l}\text { Consensus Recommendations for } \\
\text { the Management of Constipation } \\
\text { in Patients with Advanced, } \\
\text { Progressive Illness [19] }\end{array}$ & $50 \%$ & $19 \%$ & $40 \%$ & $64 \%$ & $21 \%$ & $50 \%$ & Recommended \\
\hline $\begin{array}{l}\text { Prucalopride for the treatment } \\
\text { of chronic constipation in } \\
\text { women [20] }\end{array}$ & $56 \%$ & $39 \%$ & $33 \%$ & $72 \%$ & $58 \%$ & $50 \%$ & Recommended \\
\hline $\begin{array}{l}\text { Common views on diagnosis } \\
\text { and treatment of chronic } \\
\text { constipation with Chinese } \\
\text { medicine [21] }\end{array}$ & $17 \%$ & $8 \%$ & $11 \%$ & $56 \%$ & $23 \%$ & $0 \%$ & Not recommended \\
\hline $\begin{array}{l}\text { World Gastroenterology } \\
\text { Organisation Global Guideline } \\
\text { Constipation-A Global } \\
\text { Perspective [22] }\end{array}$ & $36 \%$ & $14 \%$ & $14 \%$ & $47 \%$ & $21 \%$ & $83 \%$ & Not recommended \\
\hline $\begin{array}{l}\text { Consensus statement AIGO/SICCR } \\
\text { diagnosis and treatment of } \\
\text { chronic constipation and } \\
\text { obstructed defecation }[23,24]\end{array}$ & $50 \%$ & $19 \%$ & $48 \%$ & $50 \%$ & $21 \%$ & $17 \%$ & Not recommended \\
\hline $\begin{array}{l}\text { Practical Treatments for } \\
\text { Constipation in Korea [25] }\end{array}$ & $44 \%$ & $11 \%$ & $16 \%$ & $39 \%$ & $21 \%$ & $33 \%$ & Not recommended \\
\hline
\end{tabular}


Table 2 Guideline score according to score on each of the domains assessed by the AGREEll instrument (Continued)

\begin{tabular}{|c|c|c|c|c|c|c|c|}
\hline $\begin{array}{l}\text { Diagnosis and Treatment } \\
\text { Guideline of Chronic } \\
\text { Constipation in China [26] }\end{array}$ & $25 \%$ & $11 \%$ & $15 \%$ & $47 \%$ & $19 \%$ & $0 \%$ & Not recommended \\
\hline $\begin{array}{l}\text { Evaluation and Treatment of } \\
\text { Functional Constipation in } \\
\text { Infants and Children: Evidence- } \\
\text { Based Recommendations From } \\
\text { ESPGHAN and NASPGHAN [27] }\end{array}$ & $81 \%$ & $25 \%$ & $66 \%$ & $83 \%$ & $23 \%$ & $38 \%$ & Recommended \\
\hline $\begin{array}{l}\text { Primary Care Management of } \\
\text { Chronic Constipation in Asia: } \\
\text { The ANMA Chronic } \\
\text { Constipation Tool [28] }\end{array}$ & $50 \%$ & $36 \%$ & $22 \%$ & $53 \%$ & $23 \%$ & $38 \%$ & Recommended \\
\hline $\begin{array}{l}\text { American Gastroenterological } \\
\text { Association Medical Position } \\
\text { Statement on Constipation [29] }\end{array}$ & $33 \%$ & $17 \%$ & $25 \%$ & $61 \%$ & $42 \%$ & $25 \%$ & Not recommended \\
\hline $\begin{array}{l}\text { Lubiprostone for treating chronic } \\
\text { idiopathic constipation [30] }\end{array}$ & $75 \%$ & $39 \%$ & $46 \%$ & $44 \%$ & $38 \%$ & $67 \%$ & Recommended \\
\hline $\begin{array}{l}\text { Emerging treatments in } \\
\text { neurogastroenterology: a } \\
\text { multidisciplinary working group } \\
\text { consensus statement on opioid- } \\
\text { induced constipation [31] }\end{array}$ & $42 \%$ & $22 \%$ & $15 \%$ & $36 \%$ & $25 \%$ & $42 \%$ & Not recommended \\
\hline Total $(\bar{X} \pm S D)$ & $51.77 \pm 18.24$ & $23.73 \pm 13.16$ & $32.23 \pm 19.24$ & $56.73 \pm 12.91$ & $29.14 \pm 13.55$ & $29.59 \pm 25.91$ & - \\
\hline
\end{tabular}

below $50 \%$. Most (63.64 \%) guidelines did not provide the information whether they received funding or not $[9-11,13,14,16-18,20-22,25,26,29]$.

\section{Overall assessment}

Guidelines were graded by the overall assessment. Only two CPGs can be strongly recommended $[11,18]$. Eight can be recommended with provisions or alterations because of the most domains scoring between $30 \%$ and $60 \%[13,14,16,19,20,27,28,30]$. The remaining 12 CPGs were labelled as 'not recommended' due to the poor domain scores $[9,10,12,15,17,21-26,31]$ (Table 2).

\section{Stratification of CPG quality}

In order to examine which factors may have impacted quality scores in the six domains, we stratified the data on the following variables (guideline area, AGREEII publication date, publication type, working group, comprehensive search or not, fund support or not, and evidence-based or not) in Table 3. We didn't find the difference in six domains quality related to publication year of AGREEII (before or after 2010). Meanwhile, guidelines published in guideline databases were significantly have a higher scores than that in journals. The scores from CPGs developed by medical societies were higher when compared with individuals for the following items: Scope \&Purpose, Stakeholders, Rigour, and Applicability. If CPGs were evidence-based, those three domains (Rigour, Applicability and Editorial independence) would have a higher scores. Apart from above, we found no differences in the rest of the comparisons.

\section{Discussion}

We conducted a comprehensive assessment of the quality of CPGs for constipation. In general, these guidelines existed many deficits. Most of the guidelines had a low score in the following (domain 2, domain 3, domain 5 and domain 6). Table 4 showed that the scores results when compared with international CPGs level [33].

According to the results, the mean score of domain 3 received only $32.23 \%$. Methods of the search and the criteria for choose evidence must be clearly described. Meanwhile, the contents of health benefits and risks, externally reviewed by experts should be provided. In order to improve the score of domain 3, particular attention should be paid in above shortcomings.

There were only 2 CPGs included guideline developing experts in the panel $[11,18]$. What's more, no patients was invited to participate in the development term. The domain 5 "applicability" have an important role in the CPGs promotion, it should provide advice and/or tools on how the recommendations can be put into practice. These low scores reflect that CPG producers remain have much work to be done to improve guideline applicability.

Lastly, the scores in the domain 6 were less than $30 \%$. Many guidelines are developed with external funding, the name of the funding body and a statement that the funding body did not influence the content of the guideline should be explicit consideration [34]. What's more, 
Table 3 Mean ( \pm SD) AGREEll scores by subgroups

\begin{tabular}{|c|c|c|c|c|c|c|}
\hline Subgroups & Scope \& Purpose & Stakeholders & Rigour & Clarity & Applicability & Editorial Independence \\
\hline \multicolumn{7}{|l|}{ Year of publication } \\
\hline$\leq 2010(n=12)$ & $57.17 \pm 15.41$ & $26.67 \pm 14.93$ & $35.92 \pm 19.77$ & $61.00 \pm 11.4$ & $32.08 \pm 16.62$ & $25.67 \pm 25.98$ \\
\hline$>2010(n=10)$ & $45.30 \pm 20.18$ & $20.20 \pm 10.53$ & $27.80 \pm 18.80$ & $51.60 \pm 13.34$ & $25.67 \pm 25.98$ & $34.30 \pm 26.39$ \\
\hline$P$ values & 0.146 & 0.249 & 0.337 & 0.096 & 0.247 & 0.451 \\
\hline \multicolumn{7}{|l|}{ Publication Type } \\
\hline Journal $(n=16)$ & $44.72 \pm 15.14$ & $18.33 \pm 7.88$ & $25.72 \pm 17.65$ & $53.94 \pm 12.35$ & $24.17 \pm 8.30$ & $24.83 \pm 22.83$ \\
\hline Database $(n=6)$ & $68.17+14.74$ & $38.5+12.93$ & $47.67+11.84$ & $62.33+14.43$ & $45.5+12.49$ & $45.17+26.63$ \\
\hline$P$ values & 0.003 & 0.01 & 0.004 & 0.239 & 0.007 & 0.134 \\
\hline \multicolumn{7}{|l|}{ Type of development group } \\
\hline Individual $(n=4)$ & $50.00 \pm 16.79$ & $22.25 \pm 6.95$ & $30.75 \pm 24.42$ & $50.00 \pm 13.29$ & $27.75 \pm 11.47$ & $26.00 \pm 18.17$ \\
\hline Medical society $(n=18)$ & $52.17 \pm 19.07$ & $24.06 \pm 14.39$ & $32.59 \pm 18.85$ & $58.22 \pm 12.74$ & $29.44 \pm 14.17$ & $30.39 \pm 27.70$ \\
\hline$P$ values & 0.829 & 0.718 & 0.896 & 0.318 & 0.808 & 0.707 \\
\hline \multicolumn{7}{|l|}{ Systematic search } \\
\hline No $(n=14)$ & $47.57 \pm 16.55$ & $19.79 \pm 10.70$ & $29.50 \pm 17.86$ & $55.43 \pm 11.51$ & $26.79 \pm 113.64$ & $22.00 \pm 27.5$ \\
\hline Yes $(n=8)$ & $59.13+20.01$ & $30.63+15.07$ & $37.00+22.5$ & $59.00+15.70$ & $33.25+13.02$ & $42.88+17.03$ \\
\hline$P$ values & 0.190 & 0.1 & 0.428 & 0.585 & 0.288 & 0.04 \\
\hline \multicolumn{7}{|l|}{ Financial } \\
\hline No $(n=14)$ & $46.08 \pm 15.97$ & $19.23 \pm 9.74$ & $25.31 \pm 18.73$ & $52.31 \pm 10.78$ & $23.77 \pm 8.63$ & $15.08 \pm 17.59$ \\
\hline Yes $(n=8)$ & $60.00 \pm 19.21$ & $30.22 \pm 15.39$ & $42.22 \pm 16.22$ & $63.11 \pm 13.68$ & $36.89 \pm 15.85$ & $50.56 \pm 21.48$ \\
\hline$P$ values & 0.46 & 0.97 & 0.51 & 0.91 & 0.10 & 0.18 \\
\hline \multicolumn{7}{|l|}{ Evidence-based } \\
\hline No $(n=13)$ & $46.08 \pm 15.97$ & $19.23 \pm 9.74$ & $25.31 \pm 18.73$ & $52.31 \pm 10.78$ & $23.77 \pm 8.63$ & $15.08 \pm 17.59$ \\
\hline Yes $(n=9)$ & $60.00 \pm 19.20$ & $30.22 \pm 15.39$ & $42.22 \pm 16.22$ & $63.11 \pm 13.68$ & $36.89 \pm 15.85$ & $50.56 \pm 21.48$ \\
\hline$P$ values & 0.09 & 0.08 & 0.04 & 0.07 & 0.04 & 0.00 \\
\hline
\end{tabular}

there should be a clearly declaration that competing interests of guideline development group members have been recorded and addressed. Therefore, conflict of interests need to be clearly stated.

There are two guidelines which we want to recommend strongly due to their high overall quality developed by Registered Nurses Association of Ontario-Professional Association (RNAOPA) [11] and an Italian guideline by the National Collaborating Centre for Women's and Children's Health (NCC-WCH) [18]. The detailed recommendations were listed in Table 5. Eight of twenty-two guidelines can be reported with provisos and alterations $[13,14,16,19,20,27,28,30]$, while the remaining 12 CPGs could not be recommended because most domain scores below $30 \%[9,10,12,15,17,21-26,29-31]$.
However, our evaluation has several limitations. First, AGREEII rarely suggest how guidelines should select topics. To be useful, guidelines should address the challenges that clinicians face in practice, but developers may exclude clinically important topics when available evidence does not meet minimum standards. Second, inclusion criteria have a language restriction (English and Chinese), language search bias might happen. Third, we used only the AGREEII instrument evaluated the CPGs other than instruments may bring some selection bias [35]. AGREEII instrument have been introduced from 2010, frankly speaking, guidelines published before 2010 did not have access to AGREEII to comply with it. Unfortunately, there is no difference when we compare the

Table 4 A comparison of domain scores between these 22 CPGs and international level (\%)

\begin{tabular}{|c|c|c|c|c|c|c|}
\hline Domain & $\begin{array}{l}\text { Scope and } \\
\text { purpose }\end{array}$ & $\begin{array}{l}\text { Stakeholder } \\
\text { involvement }\end{array}$ & $\begin{array}{l}\text { Rigor of } \\
\text { development }\end{array}$ & $\begin{array}{l}\text { Clarity of } \\
\text { presentation }\end{array}$ & Applicability & $\begin{array}{l}\text { Editorial } \\
\text { independence }\end{array}$ \\
\hline Constipation mean scores & 52 & 24 & 32 & 57 & 29 & 30 \\
\hline International mean scores & 64 & 35 & 43 & 60 & 22 & 30 \\
\hline
\end{tabular}


Table 5 The detail recommendations information of 2 highly guidelines

\begin{tabular}{|c|c|c|}
\hline Title & Items & Recommendations \\
\hline \multirow[t]{3}{*}{$\begin{array}{l}\text { Prevention of constipation in the } \\
\text { older adult population [11] }\end{array}$} & Practice Recommendations & $\begin{array}{l}\text { Assess client history (dietary fibre, medications), Physical } \\
\text { activity, Evaluate client response for ongoing interventions }\end{array}$ \\
\hline & Education Recommendation & Comprehensive education programs \\
\hline & Organization \& Policy Recommendations & Establish an interdisciplinary team \\
\hline \multirow[t]{5}{*}{$\begin{array}{l}\text { Constipation in Children and Young } \\
\text { People: Diagnosis and Management } \\
\text { of Idiopathic Childhood Constipation } \\
\text { in Primary and Secondary Care [18] }\end{array}$} & History-taking & $\begin{array}{l}\text { Stool patterns, Symptoms associated with defecation, History, } \\
\text { Timing of onset of constipation and potential precipitating } \\
\text { factors, Passage of meconium, Growth and general wellbeing, } \\
\text { Symptoms in legs/locomotor development, Abdomen, Diet } \\
\text { and fluid intake }\end{array}$ \\
\hline & Physical examination & $\begin{array}{l}\text { Inspection of perianal area: appearance, position, patency, etc.; } \\
\text { Abdominal examination; Spine/lumbosacral region/gluteal } \\
\text { examination; Lower limb neuromuscular examination including } \\
\text { tone and strength, reflexes }\end{array}$ \\
\hline & Laxatives & $\begin{array}{l}\text { Macrogols (Polyethylene glycol3350 + electrolytes), Osmotic } \\
\text { laxatives (Lactulose); Stimulant laxatives (Sodium picosulfate, } \\
\text { Bisacodyl, Senna) }\end{array}$ \\
\hline & Diet and lifestyle & $\begin{array}{l}\text { Infant formula, weaning, insufficient fluid intake; Adequate fluid } \\
\text { intake; Adequate fibre }\end{array}$ \\
\hline & Clinical investigations & $\begin{array}{l}\text { Endoscopy, Test for coeliac disease and hypothyroidism, Test for } \\
\text { coeliac disease and hypothyroidism, plain abdominal radiograph, } \\
\text { Transit studies, Rectal biopsy, Ultrasound; biofeedback; Antegrade } \\
\text { colonic enema procedure }\end{array}$ \\
\hline
\end{tabular}

six domains quality before and after 2010. We can find even if methodological requirements for CPGs are reported comply with these remains unsatisfactory. What's more, how to spread the CPGs preferable is essential for clinical practice [36]. Through above specific methodological quality analysis, which can effectively promote the development of future constipation CPGs.

\section{Conclusions}

The results find that the quality of CPGs for constipation is poor. Guideline quality may be improved if we comply with the AGREEII instrument.

\section{Competing interests}

The authors declare that they have no competing interests.

\section{Authors' contributions}

THL, DC, GJF, LN, MCL: study concept, data extraction and wrote the manuscript; GLL, CQY, GXL, LVM: statistical analysis and critical revisions of the manuscript; LN, ZWM, LJS: study concept and design study supervision; All authors have approved the final manuscript.

\section{Acknowledgment}

This work was funded by National Natural Science Foundation of China (81270006) and 12th Five-Year Major Program of Army Research (AWS12J001). We would like to thank Nadeem Anjum from Nanjing University for the language modifications.

\section{Author details}

${ }^{1}$ Department of General Surgery, Jinling Hospital, Medical School of Nanjing University, No. 305 East Zhongshan Road, Nanjing 210002, China.

2Department of Medicinal Chemistry, University of Washington, Seattle, WA, USA.
Received: 10 August 2015 Accepted: 25 April 2016

Published online: 04 May 2016

\section{References}

1. Liem O, Harman J, Benninga M, Kelleher K, Mousa H, Di Lorenzo C. Health utilization and cost impact of childhood constipation in the United States. J Pediatr. 2009;154:258-62.

2. Wong SW, Lubowski DZ. Slow-transit constipation: evaluation and treatment. ANZ J Surg. 2007:77:320-8.

3. van den Berg MM, Benninga MA, Di Lorenzo C. Epidemiology of childhood constipation: a systematic review. Am J Gastroenterol. 2006;101:2401-9.

4. Field MJ, Lohr LK. Clinical practice guidelines; directions for a new program. Washington: National Academy Press; 1990

5. Lo Vecchio A, Giannattasio A, Duggan C, De Masi S, Ortisi MT, Parola L, et al Evaluation of the quality of guidelines for acute gastroenteritis in children with the AGREE instrument. J Pediatr Gastroenterol Nutr. 2011:52:183-9.

6. Brouwers MC, Kho ME, Browman GP, Burgers JS, Cluzeau F, Feder G; AGREE Next Steps Consortium. AGREE II: advancing guideline development, reporting and evaluation in health care. CMAJ. 2010;18:E839-42.

7. Schmidt S, Follmann M, Malek N, Manns MP, Greten TF. Critical appraisal of clinical practice guidelines for diagnosis and treatment of hepatocellular carcinoma. J Gastroenterol Hepatol. 2011;26:1779-86.

8. Shrout PE, Fleiss JL. Intraclass correlations: uses in assessing rater reliability. Psychol Bull. 1979;2:420-8.

9. Locke 3rd GR, Pemberton JH, Phillips SF. American Gastroenterological Association Medical Position Statement: guidelines on constipation. Gastroenterol. 2000;119:1761-6.

10. Folden SL. Practice guidelines for the management of constipation in adults. Rehabil Nurs. 2002;27:169-75.

11. Registered Nurses' Association of Ontario. Prevention of Constipation in the Older Adult Population. (Revised). Toronto, Canada: Registered Nurses' Association of Ontario; 2005. www.rnao.org/bestpractices. Accessed 24 May 2014.

12. Bleser S, Brunton S, Carmichael B, Olden K, Rasch R, Steege J. Management of chronic constipation: recommendations from a consensus panel. J Fam Pract. 2005:54:691-8.

13. Constipation Guideline Committee of the North American Society for Pediatric Gastroenterology, Hepatology and Nutrition. Evaluation and treatment of constipation in infants and children: recommendations of the 
North American Society for Pediatric Gastroenterology, Hepatology and Nutrition. J Pediatr Gastroenterol Nutr. 2006;43:e1-13.

14. Woolery M, Bisanz A, Lyons HF, Gaido L, Yenulevich M, Fulton S, et al. Putting evidence into practice: evidence-based interventions for the prevention and management of constipation in patients with cancer. Clin J Oncol Nurs. 2008;12:317-37.

15. Larkin PJ, Sykes NP, Centeno C, Ellershaw JE, Elsner F, Eugene B; European Consensus Group on Constipation in Palliative Care. The management of constipation in palliative care: clinical practice recommendations. Palliat Med. 2008:22:796-807.

16. McKay SL, Fravel M, Scanlon C. Management of constipation. lowa City: University of lowa Gerontological Nursing Interventions Research Center, Research Translation and Dissemination Core; 2009. p. 10.51.

17. Indonesian Society of Gastroenterology (ISG). National consensus on the management of constipation in Indonesia. Acta Med Indones. 2010:43: $267-74$

18. National Collaborating Centre for Women's and Children's Health (UK) Constipation in children and young people: diagnosis and management of idiopathic childhood constipation in primary and secondary care. London: RCOG; 2010. National Institute for Health and Clinical Excellence: Guidance.

19. Librach SL, Bouvette M, De Angelis C, Farley J, Oneschuk D, Pereira JL; Canadian Consensus Development Group for Constipation in Patients with Advanced Progressive IIIness. Consensus recommendations for the management of constipation in patients with advanced, progressive illness. J Pain Symptom Manage. 2010;40:761-73.

20. Prucalopride for the treatment of chronic constipation in women. NICE technology appraisal guidance 211. https://www.nice.org.uk/guidance/ ta211.

21. China Association of Chinese Medicine. Common views on diagnosis and treatment of chronic constipation with Chinese medicine. Beijing J Tradit Chin Med. 2011;30:3-7.

22. Lindberg G, Hamid SS, Malfertheiner P, Thomsen OO, Fernandez LB, Garisch J; World Gastroenterology Organisation. World Gastroenterology Organisation global guideline: constipation-a global perspective. J Clin Gastroenterol. 2011:45:483-7.

23. Bove A, Pucciani F, Bellini M, Battaglia E, Bocchini R, Altomare DF, et al. Consensus statement AIGO/SICCR: diagnosis and treatment of chronic constipation andobstructed defecation (part I: diagnosis). World 」 Gastroenterol. 2012;18:1555-64.

24. Bove A, Bellini M, Battaglia E, Bocchini R, Gambaccini D, Bove V, et al. Consensus statement AIGO/SICCR diagnosis and treatment of chronic constipation andobstructed defecation (part II: treatment). World J Gastroenterol. 2012;36:4994-5013.

25. Park KS, Choi SC, Park MI, Shin JE, Jung KW, Kim SE, et al. Practical treatments for constipation in Korea. Constipation Study Group in Korean Society of Neurogastroenterology and Motility. Korean J Intern Med. 2012;27:262-70.

26. Chinese Medical Association of Gastroenterology Branch and Chinese Medical Association of Surgery Branch. Diagnosis and treatment guideline of chronic constipation in china. Chin J Gastroenterol. 2013;18:605-12.

27. Tabbers MM, DiLorenzo C, Berger MY, Faure C, Langendam MW, Nurko S; European Society for Pediatric Gastroenterology, Hepatology, and Nutrition; North American Society for Pediatric Gastroenterology. Evaluation and treatment of functional constipation in infants and children: evidence-based recommendations from ESPGHAN and NASPGHAN. J Pediatr Gastroenterol Nutr. 2014;58:258-74.

28. Gwee KA, Ghoshal UC, Gonlachanvit S, Chua AS, Myung SJ, Rajindrajith S, et al. Primary care management of chronic constipation in Asia: the ANMA chronic constipation tool. J Neurogastroenterol Motil. 2013;19:149-60.

29. American Gastroenterological Association, Bharucha AE, Dorn SD, Lembo A, Pressman A. American Gastroenterological Association medical position statement on constipation. Gastroenterology. 2013;144:211-7.

30. Lubiprostone for treating chronic idiopathic constipation. NICE technology appraisal guidance 318. www.nice.org.uk/guidance/ta318.

31. Camilleri M, Drossman DA, Becker G, Webster LR, Davies AN, Mawe GM. Emerging treatments in neurogastroenterology:a multidisciplinary working group consensus statement onopioid-induced constipation. Neurogastroenterol Motil. 2014;26:1386-95.

32. Makarski J, Brouwers MC, AGREE Enterprise. The AGREE Enterprise: a decade of advancing clinical practice guidelines. Implement Sci. 2014;9:103.
33. Alonso-Coello P, Irfan A, Solà I, Gich I, Delgado-Noguera M, Rigau D, et al. The quality of clinical practice guidelines over the last two decades: a systematic review of guideline appraisal studies. Qual Saf Health Care. 2010:19:e58.

34. Choudhry NK, Stelfox HT, Detsky AS. Relationships between authors of clinical practice guidelines and the pharmaceutical industry. JAMA. 2002; 287:612-7.

35. Brouwers MC, Kho ME, Browman GP, Burgers JS, Cluzeau F, Feder G, et al. The Global Rating Scale complements the AGREE II in advancing the quality of practice guidelines. J Clin Epidemiol. 2012;65:526-34.

36. Ebben RH, Vloet LC, Verhofstad MH, Meijer S, Mintjes-de Groot JA, van Achterberg T. Adherence to guidelines and protocols in the prehospital and emergency care setting: a systematic review. Scand I Trauma Resusc Emerg Med. 2013;21:9.

\section{Submit your next manuscript to BioMed Central and we will help you at every step:}

- We accept pre-submission inquiries

- Our selector tool helps you to find the most relevant journal

- We provide round the clock customer support

- Convenient online submission

- Thorough peer review

- Inclusion in PubMed and all major indexing services

- Maximum visibility for your research

Submit your manuscript at www.biomedcentral.com/submit

) Biomed Central 